\title{
DATACION POR TERMOLUMINISCENCIA DE LA ARQUITECTURA DE LADRILLO. EL CASO DE MERIDA, RESULTADOS Y PROBLEMATICA
}

\author{
C. Blasco (1), R. DurÁn (1), M. Bendala (1), \\ P. Benéttez (2), J. G. Arribas (3), A. Millán (3) \\ y T. CaLderon (3) \\ (1) Departamento de Prehistoria y Arqeuología de la UAM \\ (2) Departamento de Química Física aplicada de la UAM \\ (3) Departamento de Geología y Geoquímica de la UAM
}

Resumen

El trabajo presenta los resultados obtenidos en los análisis de Termoluminiscencia realizados sobre muestras de ladrillos utilizados en la construcción de algunos de los momumentos más significativos de la Mérida romana como son el Teatro, el anfiteatro y el acueducto. Así mismo se exponen los problemas que, para análisis de este tipo, acarrea una arquitectura en la que se combina el aparejo de ladrillo con el granito.

Palabras clave: Termoluminiscencia. Datación. Arquitectura romana.

\section{Summary}

We present in this work the Thermoluminescence analysis of some bricks for dating the roman monuments of Emerita Augusta. Also we study the problems derived by the presence of granite.

Key words: Thermoluminescence. Dating. Roman architecture.

\section{INTRODUCCION}

La puesta en marcha de un laboratorio de Termoluminiscencia aplicado a la datación del Patrimonio arqueológico dentro del Proyecto de Investigación PB87- C009- C02- 01-02 subvencionado por la 
DIGICYT, tenía como finalidad abrir las vías que este método permite tanto para la datación, como para la autentificación de restos arqueológicos (RUBIO, I. y BLASCO, C., 1993).

Una de estas líneas posibles es la datación de ladrillos utilizados como material de construcción de monumentos, tanto arqueológicos, como histórico-artísticos en general. Ya que, aunque este material arquitectónico es empleado, de forma habitual, en edificios históricos que pueden ser datados por otros medios, especialmente a través de todo tipo de fuentes literarias y, muy particularmente, a partir de los documentos epigráficos y de los materiales muebles que suelen apoarecer asociados a los cimientos o amortizados entre los materiales de construcción, resulta muy frecuente que los monumentos hayan sido objeto de diversas reparaciones y reedificaciones o, incluso, que hayan sido rehechos en su totalidad, sin que existan noticias de estos acontencimientos.

Como experimentación de esta línea de datación se seleccionó el conjunto monumental de Mérida donde el ladrillo ha sido utilizado de manera muy general y de donde contamos con una importante documentación que puede servir de orientación para contrastar los resultados de los análisis de termoluminiscencia. Por otra parte, los monumentos de Mérida han sido uno de los objetivos de estudio del Proyecto de Investigación "Técnicas arquitectónicas de la Hispania romana" (PR83-2928) subvencionado por la DIGICYT, dentro de cuyo marco se inscribe también este trabajo.

\section{LA ELECCION DEL YACIMIENTO}

La importancia de Augusta Emerita en época romana es reconocida por todos. Desde el momento mismo de su fundación — tradicionalmente fijada en el 25 a.C. - como resultado de la reorganización administrativa de Augusto en Hispania, su rango fue el de capital de la nueva provincia Lusitania y como tal fué acondicionada y construída. Esta relevancia se dilata a través de los siglos y así, con la nueva reforma territorial y administrativa de Diocleciano, en los albores del siglo IV, se convierte en la sede del vicarius Hispaniarum. Su notoriedad en época visigoda está ampliamente avalada tanto por las fuentes escritas como por los innumerables restos arqueológicos que se han encontrado.

Los colosales monumentos de época romana que han llegado hasta nuestros días son muchos: dos puentes, uno imponente sobre el Guadiana (Lám. I, b)y otro, más modesto, sobre el Albarregas; tres grandes acueductos que, de manera escalonada en el tiempo y, a veces, de manera coetánea, garantizaban el abastecimiento de agua a la ciudad; un teatro (Lám. I) y un anfiteatro (Lám. II, a) de considerables dimensiones proporcionaban un lugar de reunión a la población; dos foros, uno de carácter local y otro, de mayor tamaño y suntuosidad, para resolver toda la administración central de la provincia. No podemos dejar de mencionar su extensa muralla, el muro de contención de avenidas del río Guadiana, el templo de culto imperial (conocido como Templo de Diana), con toda probabilidad incluído en el proyecto del foro local; el posible nymphaeum que se alzaba junto a la entrada del acueducto de los Milagros en la colonia; o el imponente arco honoríico, denominado por los emeritenses como "Arco de Trajano".

Así pues, con todo este ingente material, Mérida se convierte en punto de referencia obligado para cualquier arqueólogo o historiador de la Antigüedad Hispana y prueba de ello son los abundantes trabajos que sobre esta ciudad monumental se han realizado. Sin embargo, en todos estos estudios, llegada la hora de proporcionar una datación absoluta para cualquiera de los edificios mencionados, resulta que 
todas las cronologías están elaboradas con base en los hallazgos epigráficos o numismáticos asociados a los monumentos y, en los casos en que la construcción carece de ellos, se data únicamente por paralelismos con otros tipos arquitectónicos bien fechados, v.gr. como los de Roma.

Por todo ello, al acometer modernamente la investigación de estos monumentos desde una nueva óptica basada en el análisis de las técnicas constructivas y de los detalles arquitectónicos, se pone de relieve que en la mayoría de ellos existe un marcado desfase entre la data que se extrae del análisis constructivo y el dato que proporciona la epigrafía y/o la numismática. Del mismo modo, no siempre podemos contar con la información, más o menos exacta, que aportan las fuentes documentales para aquilatar la cronología de las distintas restauraciones o remodelaciones que sufre un edificio a lo largo de sus etapas de utilización, que en muchos edificos emeritenses abarca incluso más de cinco siglos sucesivos.

En consecuencia, se emprendió la labor de indagar las posibilidades que la datación por Termoluminiscencia (TL) podía aportar a algunos edificios emeritenses, a través del análisis de los ladrillos utilizados en la construcción inicial del edificio o de alguna de sus fases subsiguientes. De esta manera, los resultados obtenidos mediante $T L$ podían ayudar a puntualizar cronologías y a esclarecer otras cuestiones más complejas, también de carácter temporal. Con estos presupuestos se pretendía además de en riquecer y perfilar con mayor nitidez datos fundamentales para la Historia de Mérida, desbrozar aspectos tan importantes para la Arquitectura Hispanorromana como la introducción del uso de ladrillo en esta zona, momento de mayor difusión, etc. y poder establecer, con datos absolutos, que existen notables diferencias en este aspecto con la capital del Imperio. Este hecho ya lo han puesto en evidencia otros invesgadores: los datos de Roma no puede ser traspolados a la península Ibérica. Por ello, a partir de este trabajo también quedaría comprobado a través de métodos físicos, como lo es la TL.

Como punto de partida se tomaron en consideración acontecimientos históricos emeritenses bien conocidos, de manera que permitiesen calibrar bien el método y se procedió a emprender el paso siguiente.

\section{EXPERIMENTAL}

Toma de muestras. Para el estudio por TL del conjunto monumental de Mérida se recogieron un total de 11 (once) muestras representarivas de varios edificios emeritenses: Teatro y Aula de Culto Imperial, Anfiteatro y Acueducto de los Milagros. (Para su exacta localización, véase Fig. 1, 2 y 3.)

El teatro de Mérida está construído con un núcleo de opus caementicium, y revestido con una magnífica fábrica de sillares de granito (opus quadratum). En toda la zona correspondiente tanto a la cavea, como a sus distintos vomitoria, itiner y crypta, es este el único sistema constructivo que actualmente se puede documentar, aunque queda constancia por grabados antiguos de que las bóvedas que cubrían las escaleras de acceso a las caveae superiores se cubráin con bóvedas latericias.

En la fachada principal, por la parte nororiental, se documenta que en fechas muy posteriores a la erección del hemiciclo (Durán Cabello, en prensa) se procedió al adosamiento de un nuevo espacio de uso teatral: una gran sala, la versura. 


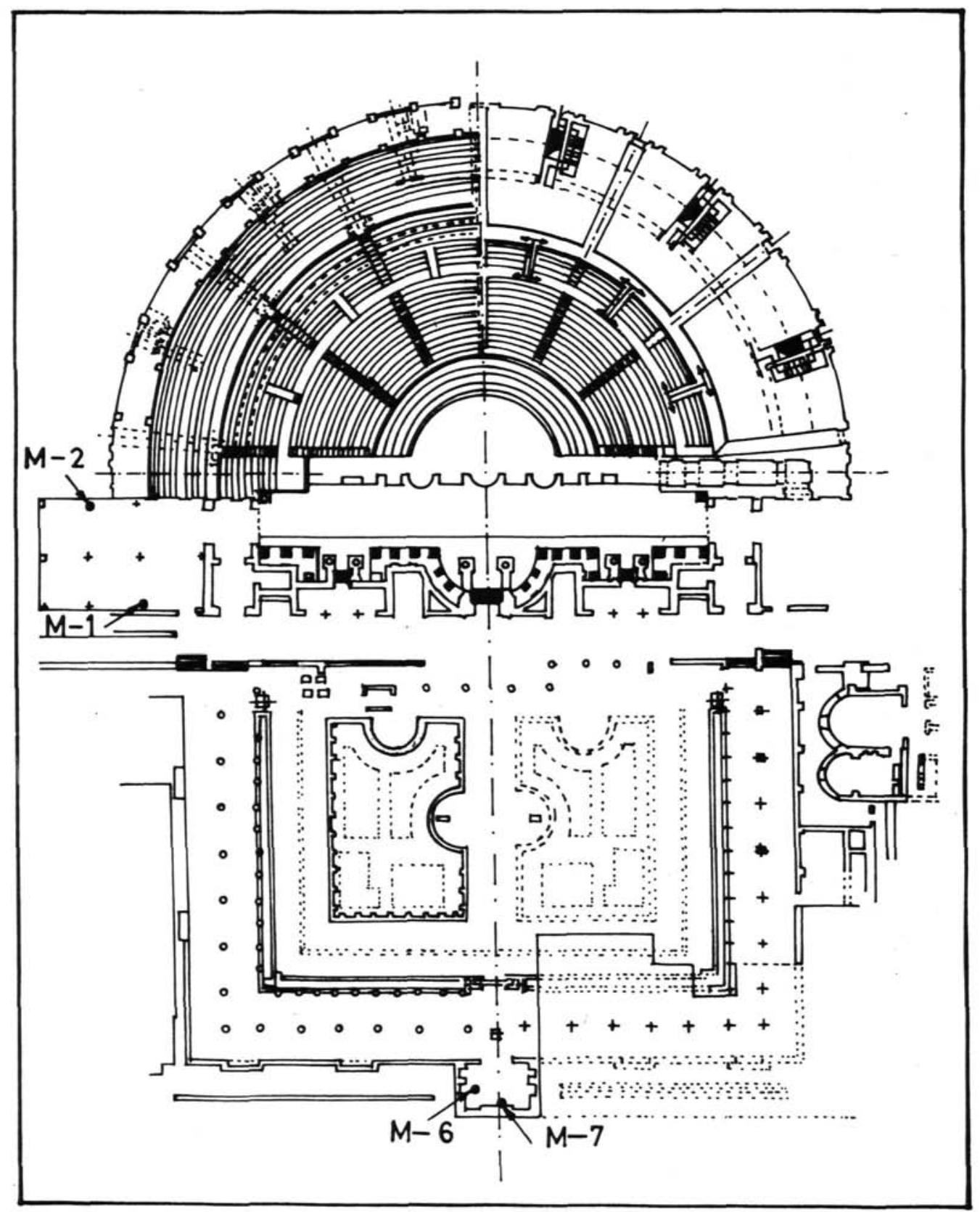

Lamina I. Teatro: M-1: Sala de la Versura. M-2: Sala de la Versura. M-6: Hornacina del cuerpo central del Aula Culto Imperial. M-7: Hornacina del ángulo noroeste del Aula Culto Imperial 
Este nuevo ambiente está construído en su casi totalidad con ladrillos. De allí se extrajeron algunas de las muestras estudiadas, de zonas no expuestas a la radiación solar (en áreas parcialmente ocultas por columnas de granito, distantes no más de $20 \mathrm{~cm}$. de esta piedra) y a una profundidad de ca. $10 \mathrm{~cm}$. con respecto a la superficie del paramento. Se tomaron las siguientes:

M-1 Muestra de ladrillo del muro norte.

M-2 Muestra de ladrillo del muro sur.

Este monumento se localiza inmediatamente al este del teatro. Está construído con una fábrica cuyo núcleo, al igual que ocurría en el teatro, es de opus caementicium; su cara vista está aparejada en una suerte de mampostería, u opus incertum. En las esquinas de los accesos al edifico se emplean sillares graníticos para cinchar los ángulos y conferir así más fortaleza a la construcción en las zonas teóricamente más delicadas. También aparecen cadenas de sillares, simulando pilastras en resalte enmarcadas en los contrafuertes del anfiteatro; de esta manera, se dota a la fachada de dinamicidad rompiendo la monotonía de los paños lisos.

El empleo del ladrillo se documenta en las bóvedas de los vomitorios y corredores secundarios de las escaleras de acceso a los maeniana superiores, así como en las ventanas que proporcionaban luz y ventilación a dichas escalerasiglo También está construído en ladrillo todo el balteus que delimita el pasillo distribuidor del primer maenianum. Por último, se constata la utilización de verdugadas de ladrillo en fachada como línea delimitadora entre los dos cuerpos, que por el lado exterior, constituían el edificio. (Bendala y Durán, en prensa).

Se extrajeron las siguientes muestras:

M-3 Muestra de ladrillo del primer vomitorio del sector oriental, comenzando por la gran puerta sur M-4 Muestra de ladrillo del primer vomitorio del sector oriental, comenzando por la gran puerta sur M-5 Muestra de ladrillo del balteus, junto a la salida del segundo vomitorio oriental, comenzando siempre por la gran puerta sur.

La muestra M-4 pertenecía a un momento histórico reciente: la restauración del edificio, situable hace 30 ó 35 años, lo que sirvió como test de comportamiento del sistema de TL utilizado. En cuanto a las otras dos muestras se han de precisar dos importantes cuestiones: una, que en ambos casos se extrajeron de zonas muy próximas a elementos graníticos (distancia no superior a $50 \mathrm{~cm}$.) de considerable magnitud: la pilastra de un arco fajón de la bóveda del vomitorio, por un lado, y el cinchado de la esquina en la confluencia entre vomitorio y pasillo distribuidor, ya en el graderío. La otra puntualización es que la muestra M-5 presentaba una textura mucho más fina que la M-3, lo cual pudiera ser debido a un mejor proceso de cocción y a la calidad intrínseca de los materiales.

Se trata de un espacio situado en el extremo norte del eje axial del peristilo del teatro. Posee planta rectangular, pero presenta un cuerpo central en avance en el panel principal de la habitación, sobre el que se abría una gran hornacina, posteriormente cegada con grandes sillares y cascote de relleno. En los dos lados menores, se abren sendos pares de hornacinas, hecho que no repercute en la planta. 


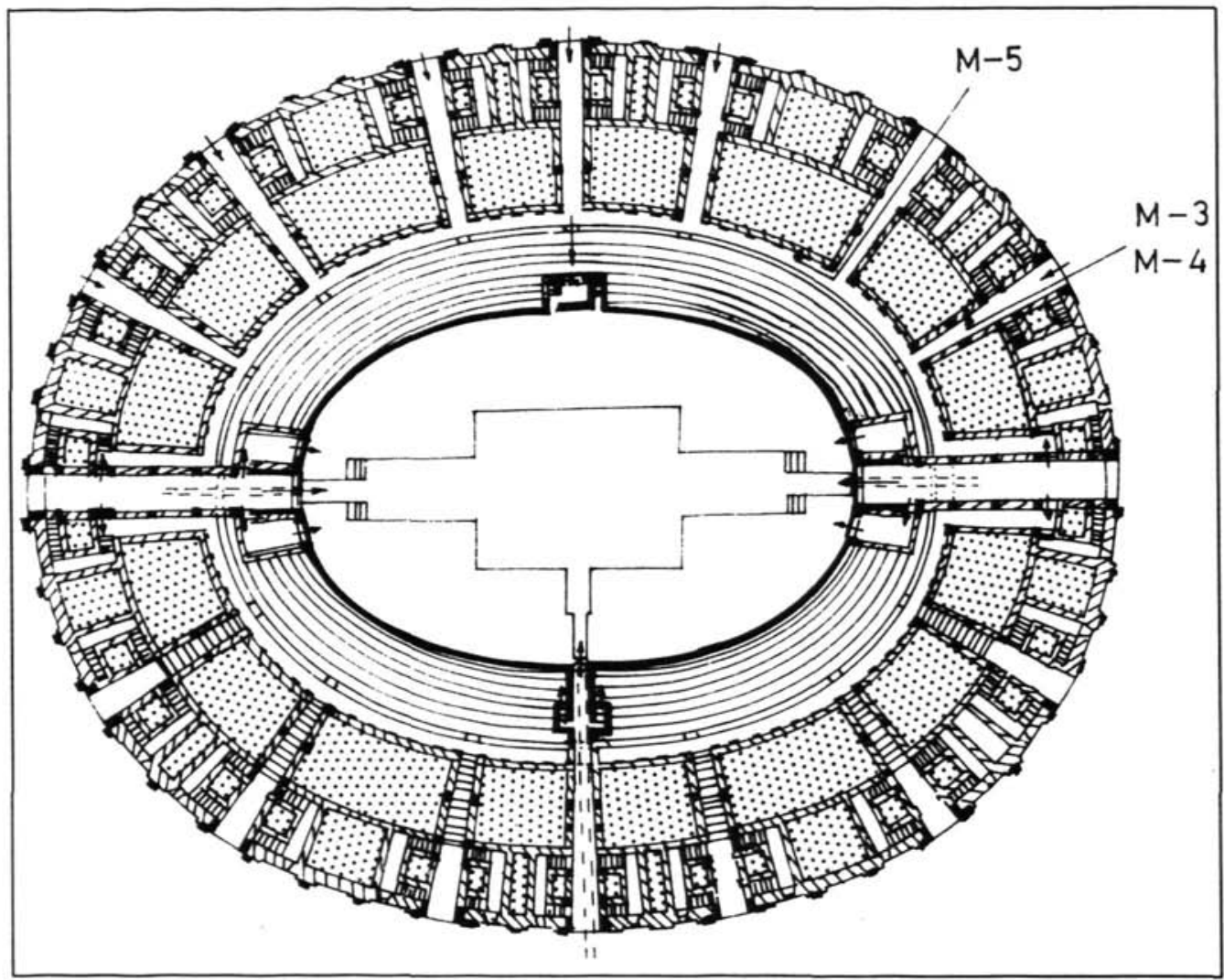

Limina II. Anfiteatro: M-3: Primer vomitorio sector oriental. M-4: Primer vomitorio sector oriental. M-5: Salida del segundo vomitorio sector oriental

Está aparejado con una fábrica mixta, atendiendo a sus materiales, pero la técnica que predomina es el opus incertum en el que se observa un importante componente de material latericio, como elemento enrasador. La otra técnica es el opus testaceum que se concentra, fundamentalmente, en el panel central adelantado y en el recrecimiento de las otras cuatro hornacinas así como en las jambas del acceso a la sala.

En este lugar se tomaron dos muestras, pertenecientes, con toda probabilidad, a dos momentos de construcción diferentes. Las muestras son:

M-6 Muestra de ladrillo de la hornacina del cuerpo central avanzado

M-7 Muestra de ladrillo de la hornacina noreste (NE) del Aula de culto.

Como en los casos anteriores, debemos puntualizar que también aquí se escogieron zonas lo más resguardadas posible de la insolación y, en ambos casos, se encontraban muy proximas a sillares graníticos incluídos en la fábrica (en torno a $50 \mathrm{~cm}$.). 


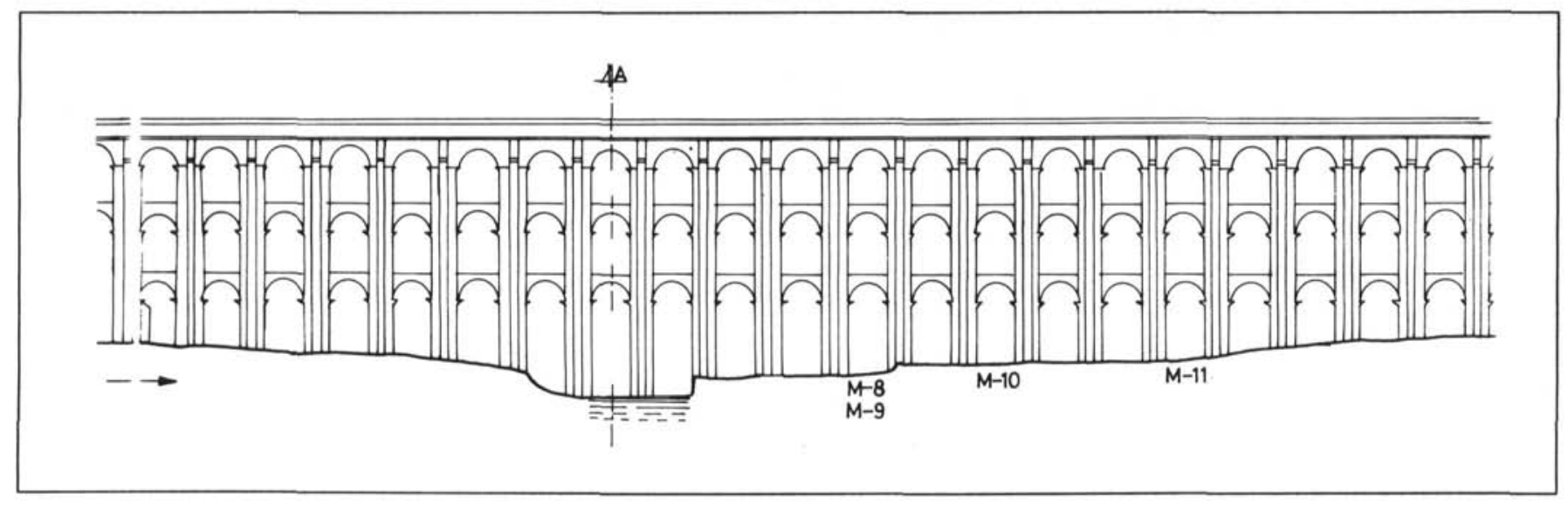

LAmina III. Acueducto de los Milagros: M-8: Ladrillo pilar n. ${ }^{\circ}$ 23. M-9: Granito pilar n. ${ }^{\circ} 23$. M-10: Ladrillo pilar n. ${ }^{\circ}$ 21. M-11: Ladrillo pilar n. 18 
Es el tercer acueducto de la colonia romana. Su caput aquae se halla en el pantano de La Albuera, también conocido como Proserpina, a 8' $5 \mathrm{~km}$. de Mérida. Su recorrido es largo y sinuoso y parece innecesario en muchos tramos, pero se desarrolla de esta manera para abaratar y agilizar los trabajos así como evitar, en lo posible, obras subterráneas y en altura, arcuationes. Es en esta última parte, las arcuationes junto a la entrada de la ciudad, donde la obra de ingeniería se convierte en monumento, al desarrollar una arquería triple - en altura - con casi cuarenta pilares de sustentación, para salvar el arroyo Albarregas.

La técnica constructiva que se plasma en esta zona consiste en pilares cuadrados con núcleo de caementicium, cuyos caementa son en un considerable porcentaje, de naturaleza granítica. Por la parte externa están revestidos con sillares de granito, en un alto porcentaje reutilizados, que cada cierta distancia se ven animados con la presencia de verdugadas de ladrillo, compuestas por cinco hiladas.

Por la parte exterior de los pilares aparecen estribos de plan rectangular que se adosan a ellos como una cuńa, ya que disminuyen su proyección con la altura. Dichos estribos están construídos en su parte inferior con sillares de granito, de muy buena factura. En altura aparecen sillarejos en vez de sillares así como la verdugada de ladrillo que se aprecia en el pilar propiamente dicho.

Los arcos son de medio punto y su luz es la de separación entre pilares; están confeccionados con ladrillo, aunque el arco central, justo sobre el Albarregas, está ejecutado todo él con dovelas graníticas.

En este área descrita se tomaron un total de cuatro muestras, de las que tres eran ladrillos de las verdugadas que jalonan los pilares, y la cuarta es un trozo de granito perteneciente a uno de esos sillares. En este caso los ladrillos están en contacto directo con el aparejo granítico. Las muestras extraídas del acueducto son:

M-8 Muestra de ladrillo del pilar $n .^{\circ} 23$, intradós norte.

M-9 Muestra de granito del pilar $n .^{\circ} 23$, intradós norte.

M-10 Muestra de ladrillo del pilar $n .^{\circ} 21$, intradós sur.

M-11 Muestra de ladrillo del pilar $n .^{\circ} 18$, zona este (estribo oriental).

\section{TRATAMIENTO DE LAS MUESTRAS}

En todas las muestras estudiadas fué eliminada la capa superficial de aproximadamente $5 \mathrm{~mm}$. de espesor, recogiéndose a continuación un peso de cada muestra de alrededor de 2 gramos, mediante un raspado cuidadoso y lento con la ayuda de una espátula fina. Seguidamente se realizó una separación de tamaño de grano, entre 1-8 $\mu \mathrm{m}$, mediante suspensión de la muestra en acetona, en un bańo de ultrasonido. El material así obtenido fué depositado seguidamente en pequeños discos de acero inoxidables de $1 \mathrm{~cm}$ de diámetro, proceso para el cual se siguió el procedimiento establecido por Zimmerman (1971) y Aitken (1985).

Con el fin de evitar una posible respuesta termoluminiscente procedente de los discos de acero inoxidable, los mismos fueron limpiados previamente con tricloroestileno. 


\section{MEDIDAS DE TERMOLUMINISCENCIA}

En la forma expuesta anteriormente fueron preparados 24 discos de cada una de las muestras estudiadas, los cuales se mantuvieron durante tres días en una estufa a temperatura controlada de $50^{\circ} \mathrm{C}$. De éstos, cuatro muestras fueron urilizadas para las medidas de $\mathrm{Tl}$ natural y comportamiento supralinear , otros 12 para la medida de respuesta de TL inducida por dosis b crecientes (3.2-19.8 mGy), suministradas por una fuente de $\mathrm{Sr}-90(0.1652 \mathrm{mGy} / \mathrm{seg})$ y finalmente cuatro discos para las medidas de TL inducidas por dosis a crecientes (1.8-3.6-5.4-10.8 mGy), suministradas por una fuente de Am-24 1 (0.0301 $\mathrm{mGy} / \mathrm{seg}$ ).

A continuación cada muestra fué calentada a temperatura ambiente hasta $500^{\circ} \mathrm{C}$ con una velocidad de calentamiento de $10^{\circ} \mathrm{C} /$ seg en atmosféra de $\mathrm{N} 2$ de alta pureza.

Las medidas de TL fueron obtenidas con ayuda de un sistema modelo Ris TL-DA-10, provisto de un tubo fotomultiplicador modelo $9635 \mathrm{QA} / 1 / 824$, suministrado por "Products for Research, Inc.", acoplado a un ordenador Olivetti M-250, donde las respuestas de TL de cada una de las muestras fueron almacenadas y posteriormente analizadas.

La radiación procedente del cuerpo negro fue reducida mediante la utilización de un filtro coloreado, modelo Corning de $44 \mathrm{~mm}$ de diámetro.

\section{DOSIS ANUALES AMBIENTALES}

Las concentraciones de $\mathrm{U}$ y Th, tanto de las muestras como del material circundante a las mismas, fueron determinadas mediante la utilización de un contador de centelleo sólido (Zns), marca AeDi, desarrollado por la Universidad de Milán y basado en la utilización de una unidad de coincidencia de tiempos, que permite ajustar una amplitud de ventana temporal de $100-400 \mathrm{~ms}$, con un retraso entre cuentas de 2,5-20 ms, lo cual permitía reconocer las desintegraciones que provenían unicamente de la cadena radiactiva del Th-232 y discriminarlas de las originadas en las cadenas del U-235 y U-238.

Las contribuciones a la dosis anual que provienen del K-40 presente en las muestras fueron establecidas mediante contaje de radiación b con un contador Geiger-Müller, modelo E-16 y desarrollado en el Centro de Investigaciones Energéticas Medioambientales y Tecnológicas de Madrid (CIEMAT).

Las contribuciones debidas a la radiación cósmica fueron establecidas mediante la utilización de un monitor de radiación gamma marca BICRON, modelo micro-analyst, provisto de un centelleador de $\mathrm{NaI}(\mathrm{Tl})$.

\section{RESULTADOS Y DISCUSION}

Para comenzar se realizó un test de comportamiento del sistema, mediante la utilización de una muestra de edad conocida (M-4), procedente de la restauración del anfiteatro realizada en la década de los 50 de la presente centuria. Los resultados obtenidos son mostrados en la tabla I (M-4), existiendo un cierto desfase entre la edad propuesta y la real ya que para una antigüedad de unos 34 años se ha obtenido 
una datación de $56+14$, un problema de envejecimiento que posiblemente afecta a la mayoría de los resultados obtenidos por $\mathrm{Tl}$ en este yacimiento.

Las muestras M-1 y M-2 de la Sala de la Versura mostraron curvas de crecimiento similares, presentando un pico pronunciado alrededor de $350^{\circ} \mathrm{C}$ y considerablemente estable al almacenamiento de las muestras, durante una semana, en cámara oscura y a temperatura ambiente. Ambas muestras mostraban también un buen plateau en el rango de las temperaturas de $340-400^{\circ} \mathrm{C}$ y una respuesta lineal de TL con las dosis de radiación beta recibidas. En consecuencia todo ello parece indicar que la composición de las mismas no variaba notablemente.

Las muestras M-3 y M-5 del Anfiteatro mostraban curvas de crecimiemto de TL diferentes. Mientras que la muestra M-5 mostraba, en la curva de crecimiento, un solo pico de $385^{\circ} \mathrm{C}$, la muestra M-3 manifestaba la presencia de dos picos, uno a baja temperatura $\left(200^{\circ} \mathrm{C}\right)$ y otro a temperatura más alta, de alrededor de $375^{\circ} \mathrm{C}$. No obstante, ambas muestras evidenciaban una buena zona de plateau en el rango de $340-420^{\circ} \mathrm{C}$ y $340-450^{\circ} \mathrm{C}$ respectivamente. Mostrando también una buena respuesta lineal de la intensidad de TL con las dosis de radiación beta recibidas.

En principio estas diferencias de comportamiento podrían relacionarse con una diferente composición en los materiales de las muestras y por tanto en una probable diferenciación en la ejecución de las mismas.

Las muestras M-6 y M-7 del Aula de Culto Imperial presentaban curvas de crecimienmto de TL similares, con un máximo a 400 y $375^{\circ} \mathrm{C}$ respectivamente y un rango de plateau de alrededor de $350-490^{\circ}$ C. Dichas muestras se diferenciaban en el comportamiento de la intensidad de TL frente a la dosis de radiación beta recibida, que en el caso de la muestra M-6 presentaba un crecimiento sublineal, para dosis beta superiores, aproximiadamente a $5 \mathrm{~Gy}$. Dicho comportamiento sugiere un proceso de saturación de la muestra que puede ser debido a alguna anomalía en su proceso de cocción, derivado o bien de no haberse alcanzado temperaturas muy altas an el mismo $\left(>500^{\circ} \mathrm{C}\right)$ o bien a tiempos de cocción no muy prolongados. En consecuencia, para dicha muestra no fué posible establecer una cronología fiable.

Las muestras M-8, M-10 y M-11 del Acueducto de los Milagros presentaban todas ellas curvas de crecimiento de TL similares, con un pico intenso a altas temperaturas, alrededor de $380^{\circ} \mathrm{C}$ y una buena respuesta lineal de TL frente a las dosis beta recibidas.

Las dosis anuales medioambientales, para cada una de las muestras bajo estudio fueron determinadas, de acuero con Aitken (1985), a partir de los siguientes datos:

a) Determinación de las concentraciones de uranio, thorio y potasio presentes en las muestras. Para dichas concentraciones, en el caso del medio circundante, se tomaron los mismos valores que para las muestras, al tratarse de matrices exactamente iguales y por lo tanto con la misma composición, excepto en el caso de las muestras $\mathrm{M}-8, \mathrm{M}-10$ y $\mathrm{M}-11$ del Acueducto en donde al tratarse de ladrillos prácticamente en contacto con grandes bloques de granito,y al haberse comprobado un excesivo envejecimiento en los resultados que ofrecieron inicialmente estas muestras: $2070+164,2063+178$ y $2075+$ 168 , que no eran justificables históricamente, con una problemática similar a la derivada de los resultados de los análisis de los ladrillos procedentes de la restauración del Anfiteatro de 1957, se determinaron las concentraciones de dichos radionúclidos en la matriz del granito (M-9).

b) La contribución a la dosis anual, debida a la radiación cósmica, fué determinada por medida directa de esta última con un monitor portátil provisto de un centelleador sólido de $\mathrm{Na}(\mathrm{Tl})$. Observán- 
dose importantes variaciones dentro del conjunto arquitectónico, que oscilaron dentro del rango 1.3-3.3 mGy/año.

c) Los contenidos de agua de las muestras así como su contenido en agua de saturación, fueron determinados experimentalmente y efectuadas las correcciones de las dosis anuales, de acuerdo al modelo establecido por Fleming (1979).

Los resultados para todas estas contribuciones a la dosis anual, así como las obtenidas de los estudios de Termoluminiscencia son mostrados en la Tabla I.

\section{CONCLUSIONES}

De los resultados experimentales obtenidos por los estudios de TL realizados, podemos llegar a las siguientes conclusiones:

1. Las muestras correspondientes a la Sala de la Versura del Teatro de Mérida presentaban un comportamiento similar, lo cual indica que la composición de las mismas no varía notablemente. En consecuencia la edad media obtenida por TL puede establecerse en $1953+124$ años. Sin embargo, no puede dejarse de mencionar que las muestras de ladrillo sobre las que se han hecho los análisis están próximas a materiales graníticos (en torno a los 20 centímetros) cuya influencia ha podido alterar el nivel de radiación.

Dicho nivel de radiación fué medido, de forma indirecta, pero dada la problemática existente y teniendo en cuenta que el granito de esta zona tiene un contenido en thorio y uranio muy alto, sería conveniente retomar la medición de forma directa, dejando el dosímetro durante unos seis meses.

2. Las muestras correspondientes al edificio del Anfiteatro presentaban comportamientos diferenciados, lo cual podría indicar una diversidad en la composición de las muestras. Por ello, los resultados obtenidos por $\mathrm{TL}$ para las dos muestras estudiadas (M-3 y M-5) y que conducen al establecimiento de dos edades $1970+147$ y 2048+241 años respectivamente, no reflejarían tanto una diferenciación temporal, sino una respuesta distinta de los materiales que componen las dos muestras. $\mathrm{O}$, como antes indicamos, debida a su composición. Por otra parte, como en el caso de las muestras de la Sala de la Versura, también aquí los ladrillos se encontraban relarivamente próximos a bloques graníticos (en torno a unos $50 \mathrm{cms}$.) y, lo que es más importante, la segunda de las muestras se escapa del marco cronológico de la construcción del monumento, probado históricamente.

En consecuenca, nuevamente nos encontramos ante un posible caso de contaminación importante por efecto del granito e, incluso, por la propia composición de los ladrillos. Una hipótesis que parece estar avalada por el resultado de una muestra tomada en un ladrillo correspondiente a la restauración realizada entre mediados de los años 50 y principio de los ańos 60 de la presente centuria $(M-4)$ que ha proporcionado una edad de $56+14$, a todas luces mucho más alta que su edad real, en torno a los 30 ó 35 años, un problema que, además se produce en unas muestras que no estaban próximas a material granítico $y$, aunque las fechas más próximas presentan mayores dificultades para un ajuste muy riguroso, este hecho puede confirmar el supuesto que apuntábamos sobre el excesivo envejecir, "ento de todas las muestras analizadas y la necesidad de replantear un afinamiento en la posible radiación a que han estado expuestas. 


\begin{tabular}{|c|c|c|c|c|c|c|c|c|c|c|}
\hline Muestra & $E D+I(G y)$ & $\begin{array}{l}\text { U (ppm) } \\
\text { Th (ppm) } \\
\text { Ladrillo }\end{array}$ & $\begin{array}{l}U(\mathrm{ppm}) \\
\text { Th (ppm) } \\
\text { Medio }\end{array}$ & $\begin{array}{l}\mathrm{K}_{2} \mathrm{O} \\
(\%) \\
\text { Ladrillo }\end{array}$ & $\begin{array}{l}\mathrm{K}_{2} \mathrm{O} \\
(\%) \\
\text { Medio }\end{array}$ & Factor $\mathrm{K}$ & $\begin{array}{c}\mathrm{H}_{2} \mathrm{O} \\
\text { Mucstra }\end{array}$ & $\begin{array}{c}\mathrm{H}_{2} \mathrm{O} \\
\text { Saturada }\end{array}$ & $\begin{array}{l}\text { (mGy/a) } \\
\text { D. anual }\end{array}$ & $\begin{array}{l}\text { EDAD } \\
\text { (ańos) }\end{array}$ \\
\hline M-1: SALA VERSURA & $16.57+0$ & 2.05 & 2.05 & 1.94 & 1.94 & 0.42 & 5.78 & 11.56 & 8.57 & $1934+199$ \\
\hline M-2: SALA VERSURA & $18.70+0$ & $\begin{array}{l}2.31 \\
7.30\end{array}$ & $\begin{array}{l}2.31 \\
7.30\end{array}$ & 2.34 & 2.34 & 0.42 & 6.34 & 12.69 & 9,47 & $1973+149$ \\
\hline $\begin{array}{c}\text { M-3: ANFITEATRO } \\
\text { VOMITORIO CENTRAL }\end{array}$ & $19.46+0$ & $\begin{array}{l}1.65 \\
5.21\end{array}$ & $\begin{array}{l}1.65 \\
5.21\end{array}$ & 2.90 & 2.90 & 0.42 & 4.94 & 9.90 & 9.88 & $1970+147$ \\
\hline $\begin{array}{l}\text { M-4: ANFITEATRO } \\
\text { VOMITORIO CENTRAL } \\
\text { RESTAURACION }\end{array}$ & $0.68+0$ & $\begin{array}{l}2.85 \\
9.00\end{array}$ & $\begin{array}{l}2.85 \\
9.00\end{array}$ & 2.42 & 2.42 & 0.42 & 5.86 & 11.74 & 12.31 & $56+14$ \\
\hline M-5: ANFITEATRO & $23.32+0$ & $\begin{array}{l}3.05 \\
9.64\end{array}$ & $\begin{array}{l}3.05 \\
9.64\end{array}$ & 2.45 & 2.45 & 0.42 & 7.42 & 14.87 & 11.39 & $2048+241$ \\
\hline $\begin{array}{c}\text { M-6: } \\
\text { AULA CULTO IMPERIAL }\end{array}$ & - & $\begin{array}{l}2.23 \\
7.04\end{array}$ & $\begin{array}{l}2.23 \\
7.04\end{array}$ & 2.44 & 2.44 & - & - & - & - & - \\
\hline $\begin{array}{l}\text { M-7: AULA CULTO } \\
\text { IMPERAIL }\end{array}$ & $18.20+0$ & $\begin{array}{l}1.88 \\
5.94\end{array}$ & $\begin{array}{l}1.88 \\
5.94\end{array}$ & 1.43 & 1.43 & 0.67 & 6.88 & 13.79 & 9.18 & $1982+180$ \\
\hline $\begin{array}{l}\text { M-8: ACUEDUCTO } \\
\text { PILAR N.o } 3\end{array}$ & $14.97+0$ & $\begin{array}{l}1.81 \\
5.72\end{array}$ & $\begin{array}{r}3.51 \\
22.52\end{array}$ & 1.36 & 6.24 & 0.38 & 10.52 & 21.07 & 8.88 & $1685+137$ \\
\hline $\begin{array}{l}\text { M-9: GRANITO } \\
\text { PILAR N.o } 23\end{array}$ & - & - & $\begin{array}{r}3.51 \\
22.52\end{array}$ & - & - & - & - & - & - & - \\
\hline $\begin{array}{l}\text { M-10: ACUEDUCTO } \\
\text { PILAR N.० } 21\end{array}$ & $17.70+0$ & $\begin{array}{l}1.87 \\
5.91\end{array}$ & $\begin{array}{r}3.51 \\
22.52\end{array}$ & 0.94 & 6.24 & 0.51 & 10.98 & 21.98 & 10.01 & $1715+152$ \\
\hline $\begin{array}{l}\text { M-11: ACUEDUCTO } \\
\text { PILAR N.० } 18\end{array}$ & $16.85+0$ & $\begin{array}{l}2.05 \\
5.84\end{array}$ & $\begin{array}{r}3.51 \\
22.52\end{array}$ & 1.05 & 6.24 & 0.45 & 10.43 & 21.52 & 9.87 & $1706+155$ \\
\hline
\end{tabular}


3. Las muestras corespondientes al Aula de Culto Imperial del Teatro presentaron comportamientos similares. No obstante, la muestra M- 6 tuvo un comportamiento sublineal muy acentuado para dosis de irradiación beta superiores a $5 \mathrm{~Gy}$, que impedían situar adecuadamente esta muestra en el tiempo.

Los resultados obtenidos para la segunda muestra (M-7) conducían al establecimiento de la edad de $1982+180$ ańos, un resultado que nos lleva a valores muy similares a los obtenidos en una de las muestras del Anfiteatro y en las de la Sala de la Versura aunque como ellas debe de tomarse con las debidas precauciones ya que la proximidad de materiales graníticos (en torno a los $50 \mathrm{cms}$.) hace suponer que han podido estar expuestas también a unos niveles de radiación superiores a los que el dosímetro registró en una medida indirecta.

4. Las muestras correspondientes al Acueducto de los Milagros presentaban todas ellas un comportamiento muy similar. Como ya antes se ha anticipado, en una primera medición arrojaron valores de $2070+154,2063+178$ y $2075+168$, claramente anteriores a la fundación de la ciudad, por ello se volvió a hacer una nueva medición teniendo en cuenta las concentraciones de radionúclidos de la matriz del granito de los sillares próximos ya que se estimó que, al estar en contacto, pudo existir una influencia importante, con este supuesto, las fechas obtenidas fueron de $1685+137,1715+152$ y $1706+155$ respectivamente lo que significa una edad media de $1702+85$ años,. es decir 288 d.C. +85 .

En suma el proceso de análisis ha permitido reconocer la existencia de importantes dificultades en un contexto litológico tan complejo como es el de Mérida, muy particularmente por la existencia de abundantes granitos en la mayoría de las construcciones y por el alto contenido en uranio y thorio que se concentra en esos granitos locales, una circunstancia que hace aconsejable la medición de las concentraciones de uranio, thorio y potasio mediante dosímetros que recojan la información de un dilatado espacio temporal, en torno a los seis meses.

Sin embargo los resultados que arroja esta primera analíica son importantes ya que permiten albergar fundadas esperanzas eri esta línea de datación de la arquitectura que, como se desprende del caso del anfiteatro, detecta las diferentes fases de un mismo monumento o incluso problemas de derivados de la distinta composición de los ladrillos, como podría ser el caso del Anfiteatro, o de baja cocción como en el Aula de Culto.

\section{CONSIDERACIONES ARQUEOLOGICAS DE LAS DATACIONES}

Una vez obtenidos los resultados de los análisis, observamos que las dataciones que estos arrojan no se corresponden con las datas seguras que poseemos ni con las cronologías relativas que se han deducido por métodos arqueológicos.

En el caso del Teatro, el ejemplo de las dos muestras procedentes de la versura evidencian una cierta "homogeneidad", aunque extraña que paramentos realizados al unísono dentro de una obra unitaria, se distancien 39 años entre sí durante el proceso de construcción: del año 17 d.C. (aprox.) que da M-2 frente al año 56 d.C. de la M-1.

En segundo lugar, aun considerando como "aceptable" el argumento anterior, cosa un tanto forzada, no podemos pasar por alto que la versura se erige cercenando gran parte de la fachada principal, por el lado de oriente, del teatro. Este hecho extrañaría en sobremanera, ya que la obra del teatro se com- 
pleta durante el reinado de Claudio, tiempo en el que se alza tanto la fachada anular como el frente escénico $^{1}$. Por lo cual, es bastante sorprendente que se cercene la incripción bronínea de Agrippa así como las cornisas que la enmarcaban y la moldura que adornaba el frente del hemiciclo.

De igual manera, $y$ al hilo de lo que acabamos de exponer, debemos subrayar que contamos, además, con una cronología relativa aportada por los sellos que llevan los ladrillos que conforman dicha sala y que han sido objeto del presente análisis mediante TL. Dichas marcas nos conducen, a través de datos epigráficos y prosopográficos, a finales del siglo IV o comienzos del siglo $\mathrm{V} \mathrm{d.C.,} \mathrm{por} \mathrm{lo} \mathrm{que} \mathrm{queda} \mathrm{claro}$ que los resultados aportados por este análisis de TL no se aproximan en ningún momento a la realidad histórica (Durán Cabello, en prensa).

También pertenece al conjunto arquitectónico del teatro la asíllamada Aula de Culto Imperial sita al fondo del peristilo que se abre tras la scaenae frons. En este lugar se recogieron dos muestras, de las cuales sólo una pudo ser susceptible de ser analizada. La datación que arroja es del ańo $8 \mathrm{~d}$.C.. Sin embargo, esta datación se aviene mal con los resultados obtenidos del análisis de la técnica constructiva del ambiente, en el que se documentan varias fases con restauraciones y reformas, alguna de ellas de gran envergadura. Y la muestra que ha dado resultado pertenece a una fase que nosotros identificamos como pertenciente a la última remodelación del ambiente (Trillmich, W. , y Durán Cabello, R., en preparación), que debemos situar al rededor del siglo IV d.C..

Con el Anfiteatro ocurre algo similar, aunque aquí los datos se nos muestran mucho más distorsionados que en el monumento precedente. Además, como ya se ha mencionado reperidas veces a lo largo del presente escrito, es en este edificio donde se extrajo una muestra procedente de la restauración del mismo, que se llevó a cabo durante la década de los años 50, con lo que el dato servía de punto de ajuste y comprobación para calibrar el método (Menéndez-Pidal, 1955; idem, 1957-58 y 1976). Sin embargo, la fecha que ofrece el análisis es de 1934, una diferencia notabilísima que pone de manifiesto que en el caso de Mérida, el granito tiene un gran poder distorsionador por su alto contenido en thorio y uranio. De ahí que los ladrillos, ya sea por su composición (desagrasantes ricos en granito) o por la contaminación ambiental proveniente de sillares de esta naturaleza, ofrezcan unas fechas que tienden a retrotraerse muchísimo. Es decir, que muestran un envejecimiento irreal.

Como comprobación de lo que acabamos de exponer contamos con otras dos muestras, tomadas de zonas no restauradas del monumento. Así, M-3 dió como resultado una fecha del año 20 d.C. mientras que la otra, M- 5 se situa en el año $58 \mathrm{~d}$.C.. Lo primero que llama la atención es el distanciamiento cronológico existente entre una y otra, perteneciendo ambas a una misma unidad estructural, por lo que debían ser contemporáneas. Vemos que se repite, pues, el mismo caso que en la versura del teatro. Por otro lado, vemos que estas dataciones no pueden compaginarse, en ningún caso, con la fecha que se desprende de la restitución de los cuatro epígrafes (Menéndez-Pidal, 1957-58; Ramírez Sádaba, J. M., 1994, en prensa), el año $8 \mathrm{~d}$.C., ni con la fecha que se extrae del análisis arqueológico-constructivo del edificio, que nos sitúa en un momento posterior al reinado de Claudio (Bendala, M., y Durán, R., 1994 en prensa). Por tanto, queda también comprobado para el anfiteatro que las muestras están alteradas por la radiactividad ambiental por lo que deben ser revisadas con una nueva óptica.

'En la fachada semicircular se observa un trabajo muy caracteristico del periodo correspondiente a los reinados de Caligula y Claudio (Durán, 91-92, 59-64). En esta misma época, la de Claudio, debe realizarse la scaenae frons, según opinión comunicada por el Dr. W. Trillmich, al que debemos un beneficioso intercambio de ideas. 
Por último, en el caso del acueducto de Los Milagros, las dataciones son —como se verá- mucho más acordes con la realidad. En este ejemplo se aplicó una corrección a los primeros resultados obtenidos porque se puso de manifiesto el alto índice de radiactividad ambiental que desprendían los bloques graníticos que conforman el acueducto. Los primeros datos arrojaban unas dataciones que iban entre el año 85 a.C. y el 73 a.C., erróneas completamente ya que ni la misma colonia estaba fundada. Así, las dataciones definitivas, una vez efectuada la revisión, son: del año 305 d.C., para la M-8; del 275 y del 284 d.C., respectivamente, para las muestas M-10 y M-11.

En líneas generales y teniendo en cuenta el problema suscitado por los componentes graníticos, creemos que estamos en situación de decir que, en el presente ejemplo, las dataciones se ajustan —más o menos - con las evidencias arqueológicas. Efectivamente, dicho monumento, tal y conforme lo conocemos hoy es el resultado de una fuerte remodelación en la que se mezclan hiladas de sillares con verdugadas de ladrillo. Este hecho fué puesto de relieve, en primer lugar, por Hauschild (1976), quien subraya que esta técnica de construcción corresponde a una segunda fase del edificio y que el tipo empleado es muy común en el oriente del Imperio, y típico de época tardía y bizantina. Para el solar peninsular señala como paralelo de alternancia de piedra con ladrillo por "franjas", el monumento funerario de Centcelles, ya en los comedios del siglo IV. Sin embargo, este investigador se "retracta" de su hipótesis porque intenta aplicar los patrones de edilicia romana a la peninsula Ibérica, con lo que el desajuste es total. Hoy día está ya ampliamente demostrado que no podemos trasladar los resultados que Lugli (1957) obtuvo para Roma y Lacio como elemento cronológico a ningún punto del territorio hispanorromano (Roldán Gómez, 1992).

Por otro lado, tenemos la investigación realizada por Jiménez Martín en el mismo monumento (1976 y 1978). al igual que Hauschild, el constata dos momentos constructivos distintos que distingue por sus fábricas. La primera toda ella de sillares graníticos —opus quadratum — con núcleo de hormigón, se localiza en los pilares que apoyan directamente en el lecho del arroyo Albarregas, entre los cuales cabalga el único arco con dovelas graníticas. Esta fase la ubica, cronológicamente hablando, en el reinado de Trajano. La segunda, la que se caracteriza por el uso conjunto de sillares con verdugadas de ladrillo es posterior a la destrucción de la primera fase, que Jiménez la sitúa en el lapso entre Adriano y Constantino.

Llegados a este punto, vemos que las daraciones de TL se aproximan mucho más a las fechas propuestas por estos dos investigadores: entre fines del siglo III y comienzos del IV. Por tanto, creemos que es esta la vía a seguir con el resto de tomas examinadas: revisar exhaustivamente la contaminación ambiental del thorio y el uranio del granito, que tanto afecta a los otros materiales en relación con su proximidad y ańos de exposición de los ladrillos a dicha roca. La diferencia entre la datación real y la que ahora poseemos debe ser regulada con un índice corrector que puede traducirse en dos o tres siglos, aunque dado el estado actual de la investigación no podemos precisar más.

\section{BIBLIOGRAFIA}

ArTKEN, M. J. (1985): TL Dating. Academy Pressiglo London.

- (1990): Science-based Dating in Archaeology. Academy Pressiglo London.

ARRIBAS, J. G.; CaLDERON, T., y BLASCO, C. (1989): "Datación absoluta por Tl: Un ejemplo de aplicación arqueológica". Trabajos de Prehistoria, vol. 46. Madrid, pp. 231-246. 
Bendala Galán, M., y Durán Cabello, R. M. (1994): "El anfiteatrode Augusta Emerita: rasgos arquitectónicos y problemática urbanística y cronológica", Coloquio Internacional El Anfiteatro en la Hispania Romana, Mérida, 26-28 de noviembre de 1992 (en prensa).

Calderon, T.; Arribas, J. G.; Millán, A., y Blasco, C. (1988): "Servicio de datación absoluta por Termoluminiscencia y analítica de cerámicas arqueológicas en la UAM". CuPAUAM, n.* 15, Madrid, pp. 85-97.

Durán Cabello, R. M. (1991-92): "Técnicas de edilicia romana enMérida (I)", Anas, 4-5, pp. 45 ss.

DURÁN CABELLO, R. M. (E.P.): Sellos latericios procedentes del teatro romano de Mérida y depositados en el Museo Nacional de Arte Romano. Monografías Emeritenses.

FLEMING, S. J. (1970): "Thermoluminescen Dating: Refinement of the Quartz Inclusion Method", Archaeometry 12, pp. 13-30.

HausCHILD, TH. (1976): "Problemas de construcciones romanas en Mérida", Augusta Emerita, pp. 107 ss. Jiménez Martín, A. (1978): "Problemas de los acueductos emeritenses", Habis, 7, pp. 271 ss.

- (1976): "Los acueductos de Mérida", Augusta Emerita, pp. 111 ss.

LUGLI, G. (1957): La tecnica edilizia romana. Con particolar eriguardo a Roma e Lazio.

MENÉNDEZ-PIDAL ÁlvareZ, J. (1976): "Algunas notas sobre la restauración y atención prestadas a los monumentos emeritenses", Augusta Emerita, pp. 199 ss.

- (1957-58): "Restitución del texto y dimensiones de las inscripciones históricas del anfiteatro de Mérida",A.Esp.A., 30, pp. 205 ss.

- (1955): "La tribuna oriental del anfiteatro romano de Mérida", A.Esp.A., 28, pp. 292 ss.

RAMfreZ SÁDABA, J. M. (1994): "Epigrafía del anfiteatro romano de Mérida", Coloquio Internacional El Anfiteatro en la Hispania Romana, Mérida, 26-28 de noviembre de 1992 (en prensa).

ROLDÁN GOMEZ, L. (1992): Técnicas arquitectónicas en la Bética Romana. (Tesis doctoral microfichada en la Universidad Autónoma de Madrid).

RUBIO, I., y BLASCO, C. (1993): "Termoluminiscencia aplicada al Patrimonio arqueológico". Actas dos Trabalhos de Antropologia e Etnologia, Vol. XXXIII, fasc. 3-4. Porto, pp. 491-506.

TRILlmiCH, W., y DURÁN Cabello, R. M. (En preparación):"Documentación de la así llamada 'Aula de Culto Imperial'del teatro romano de Mérida".

ZIMMERNMAN, D. W., (1971): "Thermoluminescent dating using fine grain from pottery". Archaeometry, 13. pp. 29-52.

- (1973): "Introduction to basis procedures for sample preparation and thermoluminescence measurement of ceramics" PACTS, 2. pp. 1-16. 